\title{
金表面不同链长烷基硫醇自组装单分子膜表面电势的变化规律
}

\author{
高 源 徐国华 * 安 越 \\ (浙江大学化学工程与生物工程学系, 杭州 310027)
}

\begin{abstract}
摘要：从 Helmholtz 模型出发, 对生长在金表面不同链长烷基硫醇自组装单分子膜(SAM)表面电势的变化规律 进行了理论研究. 利用量子化学软件 Gaussian 03 和 MOPAC, 讨论了分子偶极矩、相对介电常数以及分子的倾 斜角对 SAM 表面电势的影响. 研究表明, 不同链长烷基硫醇 SAM 中分子的倾斜角随烷基链长度的规律性变化 是引起 SAM 表面电势变化的主要原因. 从 SAM 形成机制出发, 对金表面不同链长烷基硫醇 SAM 表面电势的 变化规律及其成因提出了新的解释.
\end{abstract}

关键词：烷基硫醇； SAM；表面电势；Gaussian 03； MOPAC 中图分类号： O647; O641

\section{Variation of Surface Potential of Alkanethiol Self-Assembled Monolayers with Different Chain Lengths on a Gold Substrate}

\author{
GAO Yuan XU Guo-Hua* AN Yue \\ (Department of Chemical and Biological Engineering, Zhejiang University, Hangzhou 310027, P. R. China)
}

\begin{abstract}
A theoretical study on the surface potential of alkanethiol self-assembled monolayers (SAMs) with different alkyl chain lengths on a gold substrate was carried out in terms of the Helmholtz model. The influences of molecular dipole moment, relative permittivity of the SAMs, and angle between the molecular chain and the substrate normal on the surface potential were systematically investigated using quantum chemical softwares Gaussian 03 and MOPAC. We found that the variation of surface potentials for the alkanethiol SAMs with different alkyl chain lengths mainly resulted from regular changes in the angles between the molecular chains and the substrate normal. We propose a new explanation for the variation in surface potentials of the alkanethiol SAMs with different alkyl chain lengths by considering the SAM formation mechanism.
\end{abstract}

Key Words: Alkanethiol; SAM; Surface potential; Gaussian 03; MOPAC

烷基硫醇 $\left(\mathrm{C}_{n} \mathrm{H}_{2 n+1} \mathrm{SH}\right)$ 能在金表面形成自组装单 分子膜 (SAM), 该特性在微电子和微器械等领域有 广泛的应用前景 ${ }^{[1-2]}$. 表面电势作为其一项重要的物 理性质而被广泛研究. 对生长在金表面不同链长的 烷基硫醇 SAM 表面电势的实验研究 ${ }^{[3-6]}$ 表明, 随着 烷基硫醇分子中烷基链上碳原子数的增加, SAM 表 面电势呈线性增长. 烷基链每增加一个碳原子, SAM 表面电势平均增加 9-14 mV. Evans 等 ${ }^{[4]}$ 认为 随着烷基链的增长, 烷基硫醇 SAM 的相对介电常 数逐渐减小, 从而导致了 SAM 表面电势的增长. 但
该解释一直受到其他学者的质疑. Taylor 等 ${ }^{[7-8]}$ 认为, 烷基链上碳原子数大于 8 的烷基 SAM 的表面电势 应该是一常数, 并指出 Evans 等在实验中观察到的 SAM 表面电势随碳链长度增加而线性增长的现象 有可能是实验测量误差或 SAM 质量差异所致. 本 文从 SAM 表面电势的 Helmholtz 模型出发, 运用量 子化学计算软件对分子偶极矩、相对介电常数以及 分子及其偶极矩与基底法线方向的夹角对 SAM 表 面电势的影响进行了理论计算和分析, 对生长在金 表面的不同链长烷基硫醇 SAM 表面电势的变化规 
律及其成因提出了新的解释.

\section{1 理论模型与计算方法}

\section{1 理论模型}

直链烷烃 SAM 可以看作是由大量具有相同大 小和取向的偶极子整齐排列形成的偶极层, 其表面 电势(即 SAM 上下层的静电势差)可以用 Helmholtz 模型 $[91$ 计算

$$
V_{\mathrm{SAM}}=\frac{\mu_{\perp}}{A \varepsilon_{\mathrm{SAM}} \varepsilon_{0}}=\frac{\mu \cos \theta}{A \varepsilon_{\mathrm{SAM}} \varepsilon_{0}}
$$

式(1)中, $\mu$ 是分子偶极矩, $\mu_{\perp}$ 是分子偶极矩在基底法 线方向上的分量, $\theta$ 是分子偶极矩与基底法线的夹 角, $A$ 是单个分子所占的基底面积, $\varepsilon_{\mathrm{SAM}}$ 是 SAM 的 相对介电常数, $\varepsilon_{0}$ 是真空介电常数. Helmholtz 模型 表明, $\mu, \theta, A$ 和 $\varepsilon_{\mathrm{SAM}}$ 都可以影响 SAM 表面电势的 大小.

\section{2 计算方法}

不同链长 $\left(\mathrm{C}_{6}-\mathrm{C}_{22}\right)$ 烷基硫醇分子的偶极矩由 Gaussian $03^{[10]}$ 计算. 早期直链烷烃衍生物的偶极矩 多采用 B3LYP/D95 方法(方法 I)进行计算 ${ }^{[11-12]}$, 但该 方法在计算精度上有所欠缺. 由于烷基硫醇分子整 体上是弱极性的共价键键合体系, 且含有较易极化 的硫原子, 理论上采用添加极化函数的中小基组计 算较为合适 ${ }^{[13]}$. 为了提高计算精度并考虑烷基硫醇 分子电子云发生极化变形的可能性, 本文还采用了 精度依次提高的两种计算方法 ${ }^{[14]}$ : B3LYP/6-31G $(d) / /$ $\mathrm{HF} / 3-21 \mathrm{G}^{*}$ (方法 II) 和 B3LYP/6-31G $(d)$ (方法 III), 对 不同链长烷基硫醇分子的偶极矩进行计算, 以期得 到更加可靠的计算结果.

在理想的烷基硫醇 SAM 中, 分子排列紧密, 不 同链长的烷基硫醇分子在基底所占的面积是相同 的. 实验和理论研究表明, 烷基硫醇 SAM 中单个分 子所占的基底面积约为 $0.214 \mathrm{~nm}^{2[1,15]}$.

SAM 的相对介电常数可以通过 ClausiusMossotti 方程 ${ }^{[4,16-17]}$ 计算获得

$$
\frac{\varepsilon-1}{\varepsilon+2}=\frac{\alpha}{3 V \varepsilon_{0}}
$$

式(2)中, $V$ 是单个成膜分子的范德华体积, 由MOPAC 计算; $\alpha$ 是分子的电子极化率 ${ }^{[16-17]}$, 采用 MOPAC 和 Gaussian 03 分别进行计算. 需要指出的是, 长链烷 基硫醇分子的电子极化率是各向异性的, 同时由于 SAM 中的分子具有高度一致的取向性, 不同方向上 原子的排列和疏密程度不同, 导致 SAM 的相对介
电常数也具有各向异性的性质. 因此, 分子的电子极 化率 $\boldsymbol{\alpha}$ 和 SAM 的相对介电常数 $\boldsymbol{\varepsilon}$ 应采用二阶张量 (三阶矩阵)表示.

烷基硫醇分子的电子极化率张量 $\boldsymbol{\alpha}$ 的具体计 算方法如下:

(i) MOPAC 法

首先采用 BFGS 方法对分子结构进行优化, 获 得稳定的分子几何结构. 然后采用 MNDO 方法计 算分子的电子极化率张量 ${ }^{[16-17]}$, 其结果是一个对角 矩阵 $\left[\begin{array}{ccc}\alpha_{x x} & 0 & 0 \\ 0 & \alpha_{y y} & 0 \\ 0 & 0 & \alpha_{z z}\end{array}\right]$, 其中 $\alpha_{x x}, \alpha_{y y}$ 和 $\alpha_{z z}$ 分别代表分子在 三个主方向上的电子极化率分量. 分别将 $\alpha_{x x}, \alpha_{y y}, \alpha_{z z}$ 代人 Clausius-Mossotti 方程, 获得对应 SAM 的相对 介电常数张量 $\left[\begin{array}{ccc}\varepsilon_{x x} & 0 & 0 \\ 0 & \varepsilon_{y y} & 0 \\ 0 & 0 & \varepsilon_{z z}\end{array}\right]$.

(ii) Gaussian 法

分别采用上述三种计算方法(I, II, III)对分子结 构进行优化并计算分子的电子极化率. Gaussian 03 给出的分子的电子极化率张量为一个对称矩阵 $\left[\begin{array}{ccc}\alpha_{11} & \alpha_{12} & \alpha_{13} \\ \alpha_{21} & \alpha_{22} & \alpha_{23} \\ \alpha_{31} & \alpha_{32} & \alpha_{33}\end{array}\right]$, 其中, $\alpha_{12}=\alpha_{21}, \alpha_{23}=\alpha_{32}, \alpha_{13}=\alpha_{31}$. 将矩阵做 对角化处理, 同样可以得到呈对角矩阵形式的电子 极化率张量 $\left[\begin{array}{ccc}\alpha_{x x} & 0 & 0 \\ 0 & \alpha_{y y} & 0 \\ 0 & 0 & \alpha_{z z}\end{array}\right]$. 再由Clausius-Mossotti 方程 计算对应 SAM 的相对介电常数张量 $\left[\begin{array}{ccc}\varepsilon_{x x} & 0 & 0 \\ 0 & \varepsilon_{y y} & 0 \\ 0 & 0 & \varepsilon_{z z}\end{array}\right]$.

Takahagi 等 ${ }^{[16-17]}$ 指出, 在有机液体本体中分子 的排列通常是随机和无序的, 电子极化率张量在不 同方向上的分布概率几乎相同, 有机液体本体的相 对介电常数具有各向同性的性质. 此时可以采用平 均极化率, $\alpha=\left(\alpha_{x x}+\alpha_{y y}+\alpha_{z z}\right) / 3$, 代人 Clausius-Mossotti 方程计算有机液体本体的表观相对介电常数. 本文 根据 Takahagi 等的研究结论, 在获得分子的电子极 化率张量后, 采用平均极化率计算烷基硫醇本体的 表观相对介电常数 $\varepsilon$, 并与烷基硫醇本体的相对介 电常数的实验测量值进行比较, 以检验各计算方法 的准确性.

通常 SAM 中的分子不会垂直生长在基底上, 分子链轴线与基底法线方向有一定的夹角. 在三维 空间中, 描述 SAM 中分子的取向至少需要考虑三 个角度(图 1): $\theta$, 分子偶极矩 $\boldsymbol{\mu}$ 与基底法线 $\boldsymbol{Z}$ 之间 
的夹角; $\theta_{1}$, 分子偶极矩 $\boldsymbol{\mu}$ 与碳链轴向量 $\boldsymbol{C}$ 之间的夹 角; $\theta_{2}$, 碳链轴向量 $\boldsymbol{C}$ 与基底法线 $\boldsymbol{Z}$ 之间的夹角(即 分子的倾斜角). 根据 Gaussian 03 计算结果, 可确定 $\boldsymbol{\mu}=\left(\mu_{1}, \mu_{2}, \mu_{3}\right), \boldsymbol{C}=\left(c_{1}, c_{2}, c_{3}\right)$ 和 $\boldsymbol{H}=\left(h_{1}, h_{2}, h_{3}\right)$ 三个向量, 其中 $\boldsymbol{H}$ 是由碳链上连接在非末端碳原子上的两个 氢原子确定的向量(图 1). 基底法线向量未知, 设为 $\boldsymbol{Z}=\left(z_{1}, z_{2}, z_{3}\right)$.

$$
\begin{aligned}
& \theta_{1} \text { 由 } \boldsymbol{\mu} \text { 和 } \boldsymbol{C} \text { 计算 } \\
& \theta_{1}=\langle\boldsymbol{\mu}, \boldsymbol{C}\rangle=\arccos \left(\frac{\mu_{1} c_{1}+\mu_{2} c_{2}+\mu_{3} c_{3}}{\sqrt{\mu_{1}^{2}+\mu_{2}^{2}+\mu_{3}^{2}} \sqrt{c_{1}^{2}+c_{2}^{2}+c_{3}^{2}}}\right)
\end{aligned}
$$

$\theta$ 可以借助 Helmholtz 模型和 SAM 表面电势 的实测值进行计算. 假定 SAM 中的分子是垂直生 长在基底上的, $\boldsymbol{C}$ 与 $\boldsymbol{Z}$ 重合, $\theta_{2}=0$, 分子偶极矩 $\boldsymbol{\mu}$ 与 基底法线 $\boldsymbol{Z}$ 方向的夹角即为 $\theta_{1}$. 根据 Helmholtz 模 型, 此时 SAM 的表面电势为

$$
V_{\perp}=\frac{\mu \cos \theta_{1}}{A \varepsilon_{\mathrm{SAM}} \varepsilon_{0}}
$$

通过计算或实验测量获得 $\mu, \theta_{1}, \varepsilon_{\mathrm{SAM}}$ 和 $A$ 参数值, 即 可计算出 $V_{\perp}$.

实际情况下 SAM 中的分子一般都是倾斜的, 分子偶极矩 $\boldsymbol{\mu}$ 与基底法线 $\boldsymbol{Z}$ 的夹角为 $\theta$ (图 1), SAM 的实测表面电势应为

$$
V_{\text {exp }}=\frac{\mu \cos \theta}{A \varepsilon_{\mathrm{SAM}} \varepsilon_{0}}
$$

如果 SAM 表面电势的实测值已知, 由公式(4)和(5) 即可求出 $\theta$

$$
\theta=\arccos \left(\frac{V_{\text {exp }}}{V_{\perp}} \times \cos \theta_{1}\right)
$$

$\theta_{2}$ 的计算需首先确定基底法线向量 $\boldsymbol{Z}$. 已有的

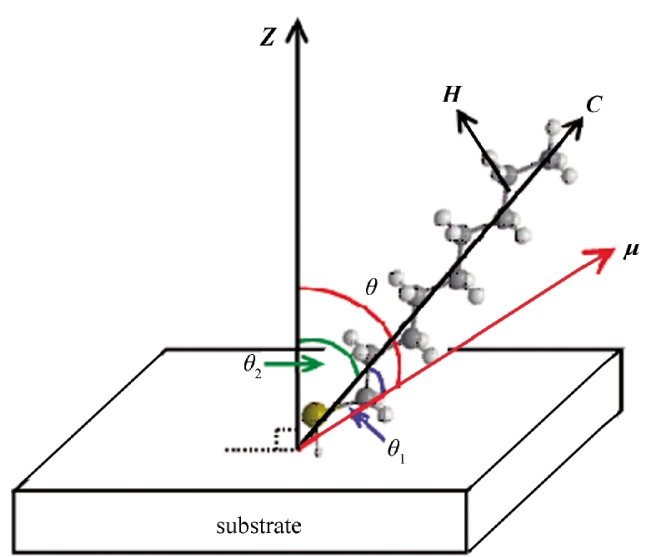

图 1 烷基硫醇 SAM 中分子取向示意图

Fig.1 Schematic diagram of the molecular orientation of alkanethiol SAM
两个约束条件是

$$
\begin{aligned}
& |\boldsymbol{Z}|=\sqrt{z_{1}^{2}+z_{2}^{2}+z_{3}^{2}}=1 \\
& \theta=\langle\boldsymbol{Z}, \boldsymbol{\mu}\rangle=\arccos \left(\frac{z_{1} \mu_{1}+z_{2} \mu_{2}+z_{3} \mu_{3}}{\sqrt{z_{1}^{2}+z_{2}^{2}+z_{3}^{2}} \sqrt{\mu_{1}^{2}+\mu_{2}^{2}+\mu_{3}^{2}}}\right)
\end{aligned}
$$

此外, Ulman 等 ${ }^{[15]}$ 对金表面烷基硫醇 SAM 中分子的 排列和取向的理论模拟研究表明,当烷基硫醇分子 绕碳链轴的自转角度为零时 (即 $\boldsymbol{Z} \perp \boldsymbol{H}$ ), 整个 $\mathrm{SAM}$ 的结构最为稳定.

$$
\boldsymbol{Z} \perp \boldsymbol{H} \Rightarrow z_{1} h_{1}+z_{2} h_{2}+z_{3} h_{3}=0
$$

由式(7-9)可确定 $\boldsymbol{Z}=\left(z_{1}, z_{2}, z_{3}\right)$, 进而求出 $\theta_{2}$

$$
\theta_{2}=\langle\boldsymbol{Z}, \boldsymbol{C}\rangle=\arccos \left(\frac{z_{1} c_{1}+z_{2} c_{2}+z_{3} c_{3}}{\sqrt{z_{1}^{2}+z_{2}^{2}+z_{3}^{2}} \sqrt{c_{1}^{2}+c_{2}^{2}+c_{3}^{2}}}\right)
$$

\section{2 结果与讨论}

\section{1 烷基链长度对分子偶极矩 $\mu$ 及其与碳链轴线 夹角 $\theta_{1}$ 的影响}

表 1 和表 2 列出了烷基链上碳原子数分别为偶 数和奇数的烷基硫醇分子的偶极矩及 $\theta_{1}$ 的计算结 果. 发现计算结果按烷基链上碳原子数的奇、偶分成 截然不同的两组, 碳原子数为偶数的烷基硫醇分子 的偶极矩及 $\theta_{1}$ 的计算结果非常接近, 同样碳原子数 为奇数的烷基硫醇分子的偶极矩及 $\theta_{1}$ 的计算结果 也很接近, 数据波动很小. 两组数据虽略有差异, 但 差距并不大, 其中烷基链上碳原子数为偶数的烷基 硫醇分子比碳原子数为奇数的烷基硫醇分子的偶 极矩及 $\theta_{1}$ 略高. 众所周知, 分子的总偶极矩是分子

\begin{tabular}{|c|c|c|c|c|c|c|}
\hline \multirow[b]{2}{*}{1} & \multicolumn{2}{|c|}{ B3LYP/D95 } & \multicolumn{2}{|c|}{ B3LYP/6-31G $(d) / / \mathrm{HF} / 3-21 \mathrm{G}^{*}$} & \multicolumn{2}{|c|}{ B3LYP/6-31G $(d)$} \\
\hline & $\overline{10^{30} \mu /(\mathrm{C} \cdot \mathrm{r}}$ & $\theta_{1} /\left(^{\circ}\right)$ & $10^{30} \mu /(\mathrm{C} \cdot \mathrm{m})$ & $\overline{\theta_{1} /\left(^{\circ}\right)}$ & $10^{30} \mu /(\mathrm{C} \cdot \mathrm{m})$ & $\overline{\theta_{1} /\left({ }^{\circ}\right)}$ \\
\hline 6 & 6.70 & 61.7 & 6.34 & 60.8 & 6.30 & 60.4 \\
\hline 8 & 6.77 & 61.7 & 6.37 & 60.7 & 6.34 & 60.4 \\
\hline 10 & 6.77 & 61.9 & 6.40 & 60.7 & 6.37 & 60.4 \\
\hline 12 & 6.80 & 61.9 & 6.40 & 60.7 & 6.37 & 60.4 \\
\hline 14 & 6.80 & 62.6 & 6.44 & 61.3 & 6.37 & 61.3 \\
\hline 16 & 6.80 & 62.6 & 6.44 & 61.3 & 6.37 & 61.3 \\
\hline 18 & 6.80 & 62.6 & 6.44 & 61.3 & 6.37 & 61.5 \\
\hline 20 & 6.80 & 61.3 & 6.44 & 61.3 & 6.40 & 61.6 \\
\hline 22 & 6.80 & 62.4 & 6.44 & 61.0 & 6.40 & 60.9 \\
\hline
\end{tabular}
中所有化学键偶极矩的矢量加和, 烷基链上亚甲基 (一 $\mathrm{CH}_{2}$ - 在空间结构上对称排列, 相邻的亚甲基所

\section{表 1 碳原子数为偶数的烷基硫醇分子 $\left(\mathrm{C}_{n} \mathrm{H}_{2 n+1} \mathrm{SH}\right)$ 的偶极} 矩 $(\mu)$ 及其与碳链轴线夹角 $\left(\theta_{1}\right)$ 的计算结果

Table 1 Calculation results of the molecular dipole moments $(\mu)$ and angles $\left(\theta_{1}\right)$ between the molecular dipole moments and axis of the carbon-chain of the alkanethiols $\left(\mathrm{C}_{n} \mathrm{H}_{2 n+1} \mathrm{SH}\right)$ with even carbon atoms $\theta_{1}$ is shown in Fig.l. 
表 2 碳原子数为奇数的烷基硫醇分子 $\left(\mathrm{C}_{n} \mathrm{H}_{2 n+1} \mathrm{SH}\right)$ 的 $\mu$ 及 $\theta_{1}$ 的计算结果

Table 2 Calculation results of $\mu$ and $\theta_{1}$ of the alkanethiols $\left(\mathrm{C}_{n} \mathbf{H}_{2 n+1} \mathbf{S H}\right)$ with odd carbon atoms

\begin{tabular}{|c|c|c|c|c|c|c|}
\hline \multirow{2}{*}{$n$} & \multicolumn{2}{|c|}{ B3LYP/D95 } & \multicolumn{2}{|c|}{ B3LYP/6-31G $(d) / / \mathrm{HF} / 3-21 \mathrm{G}$} & \multicolumn{2}{|c|}{ B3LYP/6-31G(d) } \\
\hline & $10^{30} \mu /(\mathrm{C} \cdot$ & $\overline{\theta_{1} /\left(^{\circ}\right)}$ & $10^{30} \mu /(\mathrm{C} \cdot \mathrm{m})$ & $\overline{\theta_{1} /\left(^{\circ}\right)}$ & $10^{30} \mu /(\mathrm{C} \cdot \mathrm{m})$ & $\overline{\theta_{1} /\left(^{\circ}\right)}$ \\
\hline 7 & 6.57 & 59.4 & 6.24 & 58.6 & 6.17 & 58.2 \\
\hline 9 & 6.57 & 59.9 & 6.24 & 59.0 & 6.20 & 58.8 \\
\hline 11 & 6.60 & 60.4 & 6.27 & 59.3 & 6.24 & 59.0 \\
\hline 13 & 6.60 & 61.1 & 6.27 & 60.0 & 6.24 & 60.0 \\
\hline 15 & 6.60 & 61.4 & 6.27 & 60.2 & 6.24 & 60.1 \\
\hline 17 & 6.60 & 61.5 & 6.27 & 60.3 & 6.24 & 60.2 \\
\hline 19 & 6.64 & 61.3 & 6.30 & 60.0 & 6.24 & 60.0 \\
\hline 21 & 6.70 & 61.3 & 6.30 & 60.1 & 6.24 & 60.0 \\
\hline
\end{tabular}

产生的偶极矩大小相同, 方向相反, 相互抵消 ${ }^{[7]}$. 从 理论上讲, 不同链长烷基硫醇分子的总偶极矩主要 是由分子头基和末端基团决定的, 因此烷基硫醇分 子的偶极矩及 $\theta_{1}$ 受碳链长度的影响不大. 对于烷基 链上碳原子数为偶数的烷基硫醇分子其末端基团是 乙基( $\left.-\mathrm{CH}_{2} \mathrm{CH}_{3}\right)$, 而烷基链上碳原子数为奇数的烷 基硫醇分子的末端基团是甲基 $\left(-\mathrm{CH}_{3}\right.$ ), 两末端基团 的偶极矩差异很小, 从而导致了两类分子的偶极矩 区别不大. 需要说明的是, 在表 1 和表 2 中, 虽然不 同计算方法获得的分子偶极矩的计算结果比较相 近, 但从左到右其值略有下降. 这是因为从方法 I 到 方法 III, 所用的计算方法越来越重视对分子的极化 效应的考察, 计算模拟中分子的电子云更易变形, 正 负电荷中心相互靠近, 使偶极矩有所减小.
虽然烷基链上碳原子数为偶数的烷基硫醇分子 的偶极矩略高于碳原子数为奇数的烷基硫醇分子的 偶极矩, 但计算结果表明, 不同链长的烷基硫醇分子 的偶极矩大体相同, 即随着烷基链的增长, 分子偶极 矩的大小及 $\theta_{1}$ 的变化幅度很小, 并没有出现明显的 规律性的变化. 这表明不同链长烷基硫醇分子的偶 极矩对 SAM 表面电势的影响甚微, 可以忽略.

\section{2 不同链长烷基硫醇 SAM 的相对介电常数}

表 3 是碳链长度为 $\mathrm{C}_{6}-\mathrm{C}_{22}$ 的烷基硫醇 $\mathrm{SAM}$ 的 相对介电常数张量的理论计算结果, 同时列出的还 有由平均极化率计算的烷基硫醇本体的表观相对介 电常数及其实验测量值 ${ }^{[18]}$. 结果表明, 由 MOPAC 计 算的 $\varepsilon$ 比实验测量值平均低约 $15.4 \%$, 而三种计算 方法(I, II, III)得到的 $\varepsilon$ 分别比实验值平均高出约 $3.8 \%, 5.1 \%$ 和 $6.2 \%$. 三种方法获得的计算结果与实 验值都比较接近, 也与其他研究者获得的烷基硫 醇SAM 的表观相对介电常数的结果 ${ }^{[19-21]}$ 基本一致. MOPAC 和 Gaussian 03 的计算结果都表明, 不同链 长烷基硫醇分子 SAM 的相对介电常数张量基本相 同, 其在三个主方向上的介电常数分量可视为常数, 并没有出现随着烷基链长度的变化而发生规律性的 增大或减小的趋势.

上述研究表明, 随着烷基链的增长, 烷基硫醇分 子的偶极矩, SAM 的相对介电常数几乎没有变化. 如果 SAM 中的硫醇分子都是垂直生长在基底上的,

表 3 相对介电常数张量的理论计算结果

Table 3 Calculation results of the relative permittivity tensors

\begin{tabular}{|c|c|c|c|c|c|c|c|c|c|c|c|c|c|c|c|c|c|c|}
\hline \multirow{3}{*}{$n$} & \multirow{3}{*}{$V / \mathrm{nm}^{3}$} & \multirow{2}{*}{\multicolumn{4}{|c|}{$\frac{\text { MOPAC }}{\text { MNDO//BFGS }}$}} & \multicolumn{12}{|c|}{ Gaussian 03} & \multirow{3}{*}{$\varepsilon_{\text {exp }}$} \\
\hline & & & & & & \multicolumn{4}{|c|}{ B3LYP/D95 } & \multicolumn{4}{|c|}{ B3LYP/6-31G $(d) / / \mathrm{HF} / 3-21 \mathrm{G}^{*}$} & \multicolumn{4}{|c|}{ B3LYP/6-31G $(d)$} & \\
\hline & & $\varepsilon_{x x}$ & $\varepsilon_{y y}$ & $\varepsilon_{z z}$ & $\bar{\varepsilon}$ & $\varepsilon_{x x}$ & $\varepsilon_{y y}$ & $\varepsilon_{z z}$ & $\bar{\varepsilon}$ & $\varepsilon_{x x}$ & $\varepsilon_{y y}$ & $\varepsilon_{z z}$ & $\varepsilon$ & $\varepsilon_{x x}$ & $\varepsilon_{y y}$ & $\varepsilon_{z z}$ & $\varepsilon$ & \\
\hline 6 & 0.17706 & 1.98 & 1.73 & 1.61 & 1.77 & 1.79 & 2.00 & 2.81 & 2.16 & 1.88 & 1.98 & 2.77 & 2.18 & 1.90 & 2.01 & 2.80 & 2.20 & 2.10 \\
\hline 7 & 0.19944 & 1.99 & 1.73 & 1.62 & 1.78 & 1.81 & 1.99 & 2.85 & 2.17 & 1.89 & 1.98 & 2.83 & 2.19 & 1.91 & 2.01 & 2.85 & 2.22 & 2.10 \\
\hline 8 & 0.21779 & 2.02 & 1.75 & 1.65 & 1.80 & 1.83 & 2.02 & 2.93 & 2.21 & 1.92 & 2.01 & 2.92 & 2.24 & 1.94 & 2.04 & 2.94 & 2.26 & 2.11 \\
\hline 9 & 0.24058 & 2.02 & 1.75 & 1.66 & 1.80 & 1.84 & 2.01 & 2.95 & 2.22 & 1.92 & 2.00 & 2.95 & 2.24 & 1.94 & 2.03 & 2.97 & 2.26 & 2.12 \\
\hline 10 & 0.26656 & 2.01 & 1.73 & 1.66 & 1.79 & 1.83 & 1.99 & 2.92 & 2.20 & 1.90 & 1.98 & 2.93 & 2.22 & 1.92 & 2.01 & 2.95 & 2.25 & 2.12 \\
\hline 11 & 0.28900 & 2.01 & 1.73 & 1.66 & 1.80 & 1.83 & 1.99 & 2.95 & 2.20 & 1.91 & 1.98 & 2.96 & 2.23 & 1.93 & 2.01 & 2.97 & 2.25 & 2.13 \\
\hline 12 & 0.31128 & 2.02 & 1.73 & 1.67 & 1.80 & 1.84 & 1.99 & 2.96 & 2.21 & 1.91 & 1.98 & 2.98 & 2.24 & 1.93 & 2.01 & 3.00 & 2.26 & 2.13 \\
\hline 13 & 0.33383 & 2.02 & 1.73 & 1.68 & 1.80 & 1.84 & 1.99 & 2.98 & 2.21 & 1.91 & 1.98 & 3.01 & 2.24 & 1.93 & 2.01 & 3.02 & 2.27 & 2.13 \\
\hline 14 & 0.35606 & 2.02 & 1.73 & 1.68 & 1.81 & 1.85 & 1.99 & 2.99 & 2.22 & 1.91 & 1.98 & 3.03 & 2.25 & 1.93 & 2.01 & 3.04 & 2.27 & 2.13 \\
\hline 15 & 0.37862 & 2.02 & 1.73 & 1.69 & 1.81 & 1.85 & 1.99 & 3.01 & 2.22 & 1.91 & 1.97 & 3.04 & 2.25 & 1.93 & 2.01 & 3.05 & 2.28 & 2.14 \\
\hline 16 & 0.40089 & 2.03 & 1.73 & 1.69 & 1.81 & 1.85 & 1.99 & 3.02 & 2.23 & 1.91 & 1.98 & 3.06 & 2.26 & 1.94 & 2.01 & 3.07 & 2.28 & 2.14 \\
\hline 17 & 0.42333 & 2.03 & 1.73 & 1.69 & 1.81 & 1.85 & 1.98 & 3.03 & 2.23 & 1.92 & 1.97 & 3.08 & 2.26 & 1.94 & 2.00 & 3.08 & 2.28 & \\
\hline 18 & 0.44565 & 2.03 & 1.73 & 1.70 & 1.81 & 1.86 & 1.99 & 3.04 & 2.23 & 1.92 & 1.97 & 3.09 & 2.26 & 1.94 & 2.00 & 3.10 & 2.29 & 2.15 \\
\hline 19 & 0.46805 & 2.03 & 1.73 & 1.70 & 1.82 & 1.86 & 1.98 & 3.05 & 2.24 & 1.92 & 1.97 & 3.10 & 2.27 & 1.94 & 2.00 & 3.11 & 2.29 & \\
\hline 20 & 0.49060 & 2.03 & 1.73 & 1.70 & 1.82 & 1.86 & 1.98 & 3.06 & 2.24 & 1.97 & 1.97 & 3.11 & 2.29 & 1.94 & 2.00 & 3.12 & 2.29 & \\
\hline 21 & 0.50846 & 2.05 & 1.74 & 1.71 & 1.83 & 1.84 & 1.96 & 3.00 & 2.21 & 1.93 & 1.98 & 3.16 & 2.29 & 1.95 & 2.01 & 3.16 & 2.31 & \\
\hline 22 & 0.53914 & 2.03 & 1.73 & 1.70 & 1.81 & 1.86 & 1.97 & 3.05 & 2.23 & 1.91 & 1.96 & 3.11 & 2.26 & 1.93 & 2.00 & 3.11 & 2.28 & \\
\hline
\end{tabular}


即假定 SAM 中分子倾斜角 $\theta_{2}=0$, 并认为 SAM 的表 观介电常数近似等于对应的烷基硫醇本体的表观介 电常数 ${ }^{[19-21]}$, 由式(4)可以计算出不同链长烷基硫醇 SAM 的表面电势 $V_{\perp}$ (表 4). 结果表明, 若 SAM 中分 子都是垂直生长在基底上的, 烷基硫醇 SAM 的表 面电势几乎为一定值, 三种方法计算的 SAM 表面 电势的平均值依次为 $768,741,732 \mathrm{mV}$, 数据波动基 本不超过 $4 \%$ (除 $\mathrm{C}_{7}$ 外). 可见如果不同链长烷基硫 醇 SAM 中分子的取向相同, 其表面电势的大小应 与烷基链长度无关. 显然, 这一结论与实验结果 ${ }^{[3-6]}$ 是相悖的.

\section{3 分子倾斜角对 SAM 表面电势的影响}

Evans 等 ${ }^{[4]}$ 曾使用 KPFM (Kelvin probe force microscopy)对金表面不同链长烷基硫醇 SAM 的表 面电势进行了测定. 表 4 列出了 Evans 等测定的链 长为 $\mathrm{C}_{6}-\mathrm{C}_{22}$ 的烷基硫醇 $\mathrm{SAM}$ 的表面电势 $\left(V_{\text {exp }}\right)$ 的实 测结果. 需要说明的是, 表中所列的 $V_{\text {exp }}$ 是实测表面 电势的平均值, 每个数据点的平均误差约为 $\pm(3 \%-$ $5 \%)^{[4]}$. 本文利用 Evans 等提供的实验数据, 对不同 链长烷基硫醇 SAM 中分子的倾斜角 $\theta_{2}$ 进行了估算 (表 4). 结果表明, 随着烷基链碳原子数的增加, 对应 的烷基硫醇 SAM 中分子的倾斜角有逐渐减小的趋 势, 而且随着所用计算方法精度的提高, 这一趋势愈 加明显. 不难发现, 三种方法所获得的计算结果中都 会出现几个异常数据点. 造成这一现象的原因有二: 一是 Evans 等提供的表面电势的实测数据本身就有 约 $\pm(3 \%-5 \%)$ 的误差, 在个别数据点上甚至出现明显 的异常 (如 $\mathrm{C}_{9}, \mathrm{C}_{12}$ ); 二是 $V_{\perp}$ 的理论计算值偏高, 造成 相应的角度估算值偏高 (如 $\mathrm{C}_{7}$ ). $\mathrm{C}_{7}$ 的 $V_{\perp}$ 理论值出现
异常偏高, 可能与分子两端的电荷中心逐渐靠近有 关 ${ }^{[8]}$. 具体原因尚不清楚, 有待于进一步探讨.

本文的研究表明, 不同链长烷基硫醇 SAM 中 分子的倾斜角 $\theta_{2}$ 随着烷基链上碳原子数的增加在 总体上呈明显的规律性减小, 这一结果与 Porter 等 ${ }^{[19]}$ 利用红外光谱等技术获得的不同链长烷基硫醇 SAM 分子倾斜角的研究结果是一致的. 也就是说, Evans 等报道的金表面不同链长烷基硫醇 SAM 表 面电势的变化完全可能是由 SAM 中分子倾斜角的 变化所致. 分子倾斜角出现变化的原因可以从 SAM 的形成机理进行分析和解释.

\subsection{SAM 中分子倾斜角规律性变化成因的分析}

在直链烷烃 SAM 的合成过程中存在一个临界 温度 $T_{\mathrm{c}}$ (critical temperature $)^{[1,22-24]}$, 只有当 SAM 的合 成温度低于 $T_{\mathrm{c}}$ 时, 才能获得高质量的 SAM. 当SAM 的合成温度低于 $T_{\mathrm{c}}$ 时, 成膜分子的头基与基底之间 的化学反应活性较弱, 而成膜分子烷基长链之间的 相互作用, 即内聚能(cohesive energy), 相对较强, 所 形成的 SAM 结构致密有序, SAM 中分子的倾斜角 较小. 随着 SAM 合成温度的提高, 分子头基与基底 之间的反应活性逐渐增强, 而成膜分子碳链的自由 度逐渐增大, 烷基长链之间的内聚能逐渐减小, 形成 的 SAM 结构趋于松散, 分子排列的有序度下降, SAM 中分子的倾斜角逐渐增大 ${ }^{[23]}$. 研究表明, $T_{\mathrm{c}}$ 只 是烷基链长度的函数, 随着烷基链上碳原子数的增 加 $T_{\mathrm{c}}$ 线性增长 ${ }^{[22,24]}$. Nakamura 等 ${ }^{[22]}$ 在采用电化学方 法研究金表面烷基硫醇 SAM 的电容随环境温度变 化的研究中也发现了类似临界温度的存在. 烷基硫 醇 SAM 的 $T_{\mathrm{c}}$ 同样是随着烷基链上碳原子数的增加

表 4 不同链长烷基硫醇 $\left(\mathrm{C}_{n} \mathrm{H}_{2 n+1} \mathrm{SH}\right) \mathrm{SAM}$ 中分子倾斜角 $\left(\theta_{2}\right)$ 的估算值

Table 4 Estimated results of $\theta_{2}$ of alkanethiol molecules $\left(\mathrm{C}_{n} \mathrm{H}_{2 n+1} \mathrm{SH}\right)$ with different alkyl chain lengths

\begin{tabular}{|c|c|c|c|c|c|c|c|c|c|c|}
\hline \multirow{2}{*}{$n$} & \multirow{2}{*}{$V_{\text {exp }} / \mathrm{mV}$} & \multicolumn{3}{|c|}{ B3LYP/D95 } & \multicolumn{3}{|c|}{ B3LYP/6-31G $(d) / / \mathrm{HF} / 3-21 \mathrm{G}^{*}$} & \multicolumn{3}{|c|}{ B3LYP/6-31G $(d)$} \\
\hline & & $V_{\perp} / \mathrm{mV}$ & $\theta /\left(^{\circ}\right)$ & $\theta_{2} /\left(^{\circ}\right)$ & $V_{\perp} / \mathrm{mV}$ & $\theta /\left(^{\circ}\right)$ & $\theta_{2} /\left(^{\circ}\right)$ & $V_{\perp} / \mathrm{mV}$ & $\theta /\left(^{\circ}\right)$ & $\theta_{2} /\left(^{\circ}\right)$ \\
\hline 6 & 505 & 777 & 72.1 & 23.7 & 749 & 70.8 & 22.1 & 746 & 70.4 & 22.2 \\
\hline 7 & 525 & 814 & 70.8 & 23.9 & 782 & 69.5 & 22.0 & 776 & 69.1 & 22.2 \\
\hline 8 & 550 & 767 & 70.1 & 19.6 & 737 & 68.6 & 17.9 & 732 & 68.2 & 17.8 \\
\hline 9 & 555 & 783 & 69.2 & 20.4 & 758 & 67.9 & 18.6 & 750 & 67.4 & 18.5 \\
\hline 10 & 563 & 765 & 69.7 & 18.6 & 745 & 68.3 & 17.0 & 738 & 67.9 & 16.8 \\
\hline 11 & 613 & 783 & 67.3 & 15.8 & 758 & 65.6 & 13.7 & 751 & 65.1 & 13.7 \\
\hline 12 & 600 & 766 & 68.3 & 15.5 & 741 & 66.7 & 14.0 & 735 & 66.2 & 13.7 \\
\hline 13 & 613 & 763 & 67.2 & 11.8 & 739 & 65.5 & 12.2 & 726 & 65.0 & 9.1 \\
\hline 16 & 625 & 741 & 67.2 & 11.2 & 723 & 65.5 & 10.0 & 710 & 65.0 & 9.0 \\
\hline 18 & 625 & 741 & 67.2 & 10.9 & 721 & 65.4 & 9.8 & 703 & 64.9 & 8.4 \\
\hline 20 & 650 & 770 & 66.1 & 12.1 & 713 & 64.0 & 6.8 & 700 & 63.8 & 5.3 \\
\hline 22 & 680 & 746 & 65.0 & 6.9 & 729 & 63.1 & 5.3 & 718 & 62.6 & 4.3 \\
\hline
\end{tabular}


而线性增大, 从 $\mathrm{C}_{12}$ 的 $17{ }^{\circ} \mathrm{C}$ 增大到 $\mathrm{C}_{16}$ 的 $45{ }^{\circ} \mathrm{C}$, 烷 基链每增加一个碳原子, $T_{\mathrm{c}}$ 平均增加约 $6{ }^{\circ} \mathrm{C}$.

理论上讲, 烷基链越短, 其对应的烷基硫醇 $\mathrm{SAM}$ 所要求的 $T_{\mathrm{c}}$ 越低. 但在相关的实验中 ${ }^{[4-6]}$, 所有 烷基硫醇 SAM 样品都是在相同的反应温度(通常为 室温)下合成的. 在这种情形下, 随着烷基链长度的 减短, 烷基硫醇 SAM 的合成温度与其相对应的临 界温度的差值呈线性增大, 所制备 SAM 的结构渐 趋松散, SAM 中分子的无序度增大, 分子的倾斜角 也随着烷基链长度的减短逐渐增大, 最终导致表面 电势的下降. 该推论尚需进一步实验研究的支持. 值 得注意的是, Bush 等 ${ }^{[26}$ 利用 KPFM 对 OTS (十八烷 基三氯硅烷)SAM 表面电势的实验研究表明, 不同 温度下合成的 OTS SAM 的表面电势显著不同, 并 认为这是由于合成温度的改变影响了 OTS SAM 中 分子的倾斜程度所致. Bush 等的研究结果对本文所 提的观点提供了有力的支持.

\section{3 结 论}

对烷基硫醇 SAM 表面电势影响因素的理论研 究发现, 不同链长烷基硫醇 SAM 表面电势的变化 主要是由 SAM 中分子倾斜角的变化引起的, 不同 链长的烷基硫醇分子的偶极矩及其对应 SAM 的相 对介电常数基本相同, 对 SAM 表面电势变化的影 响可以忽略. 根据 SAM 形成的反应机理, 我们认为, 造成 SAM 中分子倾斜角变化的根本原因是烷基硫 醇 SAM 的合成温度与其所对应的临界温度的差值 随着烷基链上碳原子数的减少线性增大, 导致所形 成的 SAM 的结构渐趋松散, SAM 中分子的倾斜角 逐渐增大, 最终造成 SAM 表面电势的规律性变化.

\section{References}

1 Ulman, A. Chem. Rev., 1996, 96: 1533

2 Palermo, V.; Palma, M.; Samorì, P. Adv. Mater., 2006, 18: 145

3 Kuchhal, Y. K.; Katti, S. S.; Bisways, A. B. J. Colloid Interface Sci., 1933, 1: 48

4 Evans, S. D.; Ulman, A. Chem. Phys. Lett., 1990, 170: 462

5 Lü, J.; Delamarche, E.; Eng, L.; Bennewitz, R.; Meyer, E.; Güntherodt, H. J. Langmuir, 1999, 15: 8184

6 Ichii, T.; Fukum, T.; Kobayashi, K.; Yamada, H.; Kmatsushige, K.
Nanotechnology, 2004, 15: S30

7 Taylor, D. M. Adv. Colloid Interface Sci., 2000, 87: 183

8 Taylor, D. M.; Bayes, G. F. Phys. Rev. E, 1994, 49: 1439

9 Helmholtz, H. Abhandlungen zur thermodynamik chemister vorgänge. Leipzig: Herausgegeben von Dr. MaxPlank, 1902: 51

10 Frisch, M. J.; Trucks, G. W.; Schlegel, H. B.; et al. Gaussian 03 Revision B.01. Pittsburgh, PA: Gaussian Inc., 2003

11 (a) Saito, N.; Hayashi, K.; Sugimura, H.; Takai, O.; Nakagiri, N Surf. Interface Anal., 2002, 34: 601

(b) Sugimura, H.; Hayashi, K.; Saito, N.; Nakagiri, N.; Takai, O. Appl. Surf. Sci., 2002, 188: 403

(c) Saito, N.; Hayashi, K.; Sugimura, H.; Takai, O.; Nakagiri, N Chem. Phys. Lett., 2001, 349: 172

12 Ofir, Y.; Zenou, N.; Goykhman, I.; Yitzchaik, S. J. Phys. Chem. B, 2006, 110: 8002

13 Zhang, R. Q.; Bu, Y. X.; Li, S. T.; Huang, J. H.; Han, K. L.; He, G. Z. Sci. China Ser. B-Chem., 2000, 30(5): 419 [张瑞勤, 步宇翔, 李述汤, 黄建华, 韩克利, 何国钟. 中国科学 B 辑: 化学, 2000, 30 (5): 419]

14 Foresman, J. B.; Frisch, A. Exploring chemistry with electronic structure method. 2nd ed. Pittsburgh: Gaussian Inc., 1996: 141-160

15 Ulman, A.; Eilers, J. E.; Tillman, N. Langmuir, 1989, 5: 1147

16 Yamada, K.; Saiki, A.; Sakaue, H.; Shingubara, S.; Takahagi, T. Jpn. J. Appl. Phys., 2001, 40: 4829

17 Takahagi, T.; Saiki, A.; Sakaue, H.; Shingubara, S. Jpn. J. Appl. Phys., 2003, 42: 157

18 (a) Acros Organics. Reference handbook of fine chemicals. 2006/ 2007. Geel: Acros Organics, 2006: 833-1402 (b) Aldrich Chemical Company. Handbook of fine chemicals. 2007/2008. St. Louis: Sigma-Aldrich Corporation, 2007: 812-2491

19 Porter, M. D.; Bright, T. B.; Allara, D. L.; Chidsey, C. E. D. J. Am. Chem. Soc., 1987, 109: 3559

20 Ramírez, P.; Andreu, R.; Cuesta, Á.; Calzado, C. J.; Calvente, J. J. Anal. Chem., 2007, 79: 6473

21 Smalley, J. F.; Feldberg, S. W.; Chidsey, C. E. D.; Linford, M. R.; Newton, D. M.; Liu, Y. P. J. Phys. Chem., 1995, 99: 13141

22 Aswal, D. K.; Lenfant, S.; Guerin, D.; Yakhmi, J. V.; Vuillaume, D. Anal. Chim. Acta, 2006, 568: 84

23 Limura, K.; Nakajima, Y.; Kato, T. Thin Solid Films, 2000, 379: 230

24 Brzoska, J. B.; Shahidzadeh, N.; Rondelez, F. Nature, 1992, 360: 719

25 Nakamura, T.; Aoki, K.; Chen, J. Electrochem. Commun., 2002, 4: 521

26 Bush, B. G.; DelRio, F. W.; Opatkiewicz, J.; Maboudian, R.; Carraro, C. J. Phys. Chem. A, 2007, 111: 12339 